\title{
CircASXLI Knockdown Restrains Hypoxia-Induced DDP Resistance and NSCLC Progression by Sponging miR-206
}

\author{
Liuyang $\mathrm{Yu}^{\prime}$ \\ Jing $\mathrm{Li}^{2}$ \\ Bing Peng ${ }^{\prime}$ \\ Peng Cai ${ }^{1}$ \\ Bailin Zhao' \\ Ying Chen ${ }^{3}$ \\ Hailing Zhu ${ }^{4}$ \\ 'Department of Oncology, Jing Men \\ No. 2 People's Hospital, Jingmen, Hubei, \\ 448000, People's Republic of China; \\ ${ }^{2}$ Department of Imaging, Jing Men No. 2 \\ People's Hospital, Jingmen, Hubei, \\ 448000, People's Republic of China; \\ ${ }^{3}$ Department of Radiotherapy, Jing Men \\ No. 2 People's Hospital, Jingmen, Hubei, \\ 448000, People's Republic of China; \\ ${ }^{4}$ Department of Emergency, Jing Men \\ No. I People's Hospital, Jingmen, Hubei, \\ 448000, People's Republic of China
}

Background: Non-small cell lung carcinoma (NSCLC) is a primary prevalent type of cancer in people worldwide. Cisplatin (DDP) has been widely used to treat NSCLC; however, its curative effect was restrained under hypoxia. In this study, the effects of hypoxia treatment on DDP resistance and NSCLC progression and underneath mechanism were revealed.

Methods: The expression of circular RNA ASXL1 (circASXL1) and microRNA-206 (miR206) in NSCLC tissues, cells and hypoxia-mediated NSCLC cells was determined by quantitative real-time polymerase chain reaction (qRT-PCR). The expression of proliferation, metastasis and apoptosis-related proteins, drug resistance-related protein and hypoxiainducible factor-1alpha (HIF-1 $\alpha)$ protein was detected by Western blot. The effects of circASXL1 knockdown on hypoxia-induced DDP resistance and NSCLC progression were revealed by cell counting kit- 8 proliferation (CCK-8), cell colony formation, transwell and flow apoptosis assays. RNA immunoprecipitation (RIP) assay was performed to determine whether circASXL1 could form silence-inducing complexes with miRNA. The associated relationship between circASXL1 and miR-206 was predicted by circBank online database, and identified by RNA pull-down and dual-luciferase reporter assays. The effects between circASXL1 knockdown and miR-206 downregulation on tumor growth in vivo were investigated by in vivo tumor formation assay.

Results: CircASXL1 expression was dramatically upregulated, whereas miR-206 was significantly down-regulated in NSCLC tissues, cells and hypoxia-mediated NSCLC cells as compared to control groups. CircASXL1 knockdown reversed hypoxia-mediated promotion effects on DDP resistance, cell proliferation, migration, and invasion, and inhibition impact on cell apoptosis, whereas these effects were restored by miR-206 inhibitor. Additionally, circASXL1 was found to form silence-inducing complexes with miRNA and act as a sponge of miR-206. CircASXL1 silencing downregulated HIF-1 $\alpha$ expression by controlling miR206 expression. Furthermore, circASXL1 silencing repressed tumor growth in vivo by sponging miR-206.

Conclusion: CircASXL1 knockdown inhibited DDP resistance, cell proliferation, migration and invasion, whereas induced cell apoptosis under hypoxia by associating with miR-206 in NSCLC. This study provides a new sight in treating NSCLC with DDP under hypoxia.

Keywords: hypoxia, circASXL1, miR-206, DDP, NSCLC

\section{Introduction}

Lung cancers are a class of prevalent types of cancers worldwide with morbidities about $6.7 \%$ in men and $5.9 \%$ in women. ${ }^{1}$ Non-small cell lung carcinoma (NSCLC) occupies more than $75 \%$ of lung cancers, and about $80 \%$ of these cases were 
diagnosed at an advanced stage. ${ }^{2,3}$ NSCLC is incurable because of its limited treatment and high rate of recurrence. ${ }^{4}$ Cisplatin (DDP), a platinum compound, is a first-line drug in treating cancers, including NSCLC., However, DDP sensitivity is repressed with chemoresistance progression. Therefore, revealing the regulatory mechanism of DDP resistance is necessary for NSCLC therapy.

Hypoxia is a common feature of multiply cancers. Tumor hypoxia is caused by uncontrollable cell proliferation and metabolic disorder. ${ }^{7}$ It has been revealed that hypoxia was related to drug chemoresistance. ${ }^{8,9}$ Circular RNA (circRNA) is a non-coding RNA with closed-loop structure. ${ }^{10}$ The dysregulation of circRNA is linked to cancer progression. ${ }^{11,12}$ For instance, Li et al indicated that circ_006332 contributed to bladder cancer development by regulating MYB Proto-Oncogene Like 2 expression. ${ }^{13}$ Zhang et al also revealed that circphosphoglycerate mutase 1(circ-PGAM) promoted ovarian cancer growth via sponging microRNA-542-3p (miR-5423p). ${ }^{14}$ In addition, $\mathrm{Xu}$ et al demonstrated that circ_0004771 could regulate hypoxia-mediated 5-Fluorouracil resistance to gastric cancer. ${ }^{15}$ Huang et al also illustrated that circ_0001946 repressed lung cancer development and regulated DDP resistance. ${ }^{16}$ However, the effects of circASXL1 on DDP resistance and NSCLC progression under hypoxia were unknown.

MiRNA is a non-coding RNA with about 20 nucleotides in size and regulates gene expression via binding to target gene. ${ }^{17}$ In NSCLC progression, miRNA plays a vital part. For example, miR-1183 was disclosed to suppress the proliferation and migration of NSCLC cells. ${ }^{18}$ Gao et al revealed miR-3607-3p promoted cell cycle arrest in NSCLC. ${ }^{19}$ MiR-206, a small RNA, was also indicated to suppress the proliferation and invasion of NSCLC. ${ }^{20}$ Besides, miR-206 also could modulate DDP resistance in lung cancer. ${ }^{21}$ In this experiment, the effects of miR-206 on NSCLC progression and DDP resistance under hypoxia were explored.

Herein, the expression of circASXL1 and miR-206 was determined in NSCLC tissues, cells and hypoxia-mediated NSCLC cells. The effects of circASXL1 knockdown on hypoxia-induced DDP sensitivity and NSCLC progression were revealed. Meanwhile, the mechanism of circASXL1 regulating DDP sensitivity and NSCLC progression under hypoxia was explored. Furthermore, the impacts of circASXL1 silencing on tumor growth in vivo were investigated.

\section{Materials and Methods}

\section{Tissue Collection}

Thirty-nine pairs of NSCLC tissues and healthy lung tissues were obtained from NSCLC patients from Jing Men NO.2 People's Hospital. All tissues were stored in liquid nitrogen. The Ethics Committee of Jing Men NO.2 People's Hospital agreed to this experiment. NSCLC patients signed these written informed consents.

\section{Cell Culture}

ScienCell (Carlsbad, CA, USA) provided human NSCLC cells (A549 and H1299) and human normal lung cells BEAS-2B. Cells were cultured in Dulbecco's modified Eagle's medium (DMEM; Thermo Fisher, Waltham, MA, USA) with $10 \%$ fetal bovine serum (FBS) and $1 \%$ streptomycin/penicillin (Thermo Fisher) at $37^{\circ} \mathrm{C}$ in an incubator under normoxia with $5 \% \mathrm{CO}_{2}$ and $20 \% \mathrm{O}_{2}$. For hypoxia study, cells were cultured in an atmosphere with $1 \% \mathrm{O}_{2}, 5 \% \mathrm{CO}_{2}$ and $94 \% \mathrm{~N}_{2}$.

\section{Cell Transfection}

Small hairpin RNAs targeting circASXL1 (sh-circASXL1 -1 and sh-circASXL1-2), miR-206 mimic (miR-206), miR206 inhibitor (in miR-206) and their control groups (sh$\mathrm{NC}$, miR-NC and in NC) were synthesized by GenePharma (Shanghai, China). The antagonist of miR206 (antagomiR-206) and its control (antagomiR-NC) were synthesized by Ribobio Co., Ltd. (Guangzhou, China). sh-circASXL1-1 and sh-NC were used to determine the effects of circASXL1 knockdown on hypoxiainduced NSCLC progression, DDP resistance and tumor growth. sh-circASXL1-1, miR-206 inhibitor, sh-NC and in NC were utilized to detect the impacts between circASXL1 knockdown and miR-206 inhibitor on hypoxia-induced NSCLC progression and DDP resistance. sh-circASXL1-1, sh-NC, antagomiR-206 and antagomiR$\mathrm{NC}$ were used to determine the effects between circASXL1 knockdown and miR-206 absence on hypoxiainduced NSCLC growth in vivo. Cell transfection was performed with lipofectamine 2000 (Thermo Fisher).

\section{Quantitative Real-Time Polymerase Chain Reaction (qRT-PCR)}

Collected samples were lysed with TRIzol reagent (TaKaRa, Dalian, China). RNA was extracted and cDNA was synthesized via prime Script ${ }^{\mathrm{TM}}$ RT reagent Kit (TaKaRa). For detecting the expression of circASXL1, 
18S rRNA, U6, miR-206 and GAPDH, qRT-PCR detection kit (TaKaRa) was employed with Bio-Rad IQ5 thermocycler (Bio-Rad, Hercules, CA, USA). Data were analyzed with the $2^{-\Delta \Delta C t}$ method. U6 and glyceraldehyde 3-phosphate dehydrogenase (GAPDH) were utilized as controls. The sense and antisense primers were circASXL1 5'-TAAACTGCCTGGCCGAATC-3' and 5'TCCTTCTGCCTCTATGACCTG-3'; 18S rRNA 5'GATGGTAGTCGCCGTGCC-3' and 5'-GCCTGCTG CCTTCCTTGG-3'; U6 5'-CTCGCTTCGGCAGCACA-3' and 5'-AACGCTTCACGAATTTGCGT-3'; GAPDH 5'GGTCACCAGGGCTGCTTT-3' and 5'-GGAAGATG GTGATGGGATT-3'; miR-206 5'ACACTCCAGCTGGGTGGAATGTAAGGAAGT-3 and 5'-TGGTGTCGTGGAGTCG-3'.

\section{RNase R Treatment Assay}

A549 cells and H1299 cells were collected and divided into two groups, respectively. Cells were treated with or without RNase R (3 U/ $\mu$ g; Epicentre, Madison, WI, USA) at $37^{\circ} \mathrm{C}$ for $20 \mathrm{~min}$. Then, RNA was purified with phenol, chloroform and isoamyl alcohol. CircASXL1 stability was determined by detecting circASXL1 expression by qRTPCR.

\section{Cell Localization of circASXL I}

PARISTM Kit (Thermo Fisher) was used to determine the cell localization of circASXL1. In short, cells were harvested and washed using phosphate-buffered saline buffer (PBS). Then, cells were suspended using cell fractionation buffer and incubated for $8 \mathrm{~min}$. And supernatant was carefully collected and placed into RNase-free tube. The nuclear pellet and supernatant were treated. Following circASXL1 expression in nuclear pellet and cytoplasm was analyzed by qRT-PCR. 18S rRNA and U6 were employed as controls.

\section{Cell Counting Kit-8 (CCK-8) Assay}

The cell viability and half-maximal inhibitory concentration (IC50) value of DDP were assessed by CCK-8 assay. For detecting cell viability, A549 cells and H1299 cells were seeded in 96-well plate for $16 \mathrm{~h}$. Then, cells were cultured under hypoxia or normoxia with various transfection. After $24 \mathrm{~h}, 48 \mathrm{~h}$ or $72 \mathrm{~h}, 10 \mu \mathrm{L}$ CCK-8 solution (Beyotime, Jiangsu, China) was treated, followed cells were cultured for $4 \mathrm{~h}$. The results were analyzed via detecting the absorbance at $450 \mathrm{~nm}$ with microplate reader (Thermo Fisher). For determining IC50 value of DDP, cells were treated with DDP $(1.56,3.13,6.25,12.5,25$ and $50 \mu \mathrm{M})$. The IC50 value of DDP was assessed with the above method.

\section{Cell Colony Formation Assay}

A549 cells and H1299 cells were seeded in a 6-well plate for 2 weeks ( 500 cells per well). Then, cells were washed and proliferating colonies were fixed with paraformaldehyde (Beyotime). Following colonies were stained with crystal violet (Beyotime). Colonies were photographed and cell colony-forming ability was determined by accounting colonies number. More than 50 cells per colony were considered as colonies.

\section{Transwell Assay}

Cell migration and invasion were assessed with transwell chamber without or with Matrigel (Corning, New York, Madison, USA), respectively. Shortly, A549 cells and H1299 cells were suspended with FBS-free DMEM and were seeded in upper chamber. In lower chamber, DMEM with $15 \%$ FBS was added. The supernatant was discarded after $24 \mathrm{~h}$. Cells in the lower chamber were incubated with methanol and crystal violet (Beyotime). Results were analyzed with microscope $(100 \times)$.

\section{Western Blot}

NSCLC cells were lysed with RIPA buffer (Beyotime). The protein sample was boiled and loaded by $12 \%$ sodium dodecyl sulfonate-polyacrylamide gel electrophoresis (SDS-PAGE). Protein was transferred into nitrocellulose membranes (Membrane Solutions, Shanghai, China). Bands were blocked in 5\% skim milk. Then, bands were incubated with anti-c-myc (1:1000; Abcam, Cambridge, UK), anti-E-cadherin (1:1000; Abcam), anti-Vimentin (1:5000; Abcam), anti-caspase-3 (anti-casp-3) (1:10000; Abcam), anti-multi-drug resistance gene-1 (anti-MDR1; 1:500, Abcam), anti-HIF-1 $\alpha$ (1:10000; Abcam) and antiGAPDH (1:10000; Abcam). Following proteins were reacted with secondary antibody labeled with horseradish peroxidase (1:1000; Abcam). Proteins were visualized with enhanced chemiluminescence (KeyGen, Nanjing, China). GAPDH was used as a reference.

\section{Flow Cytometry Analysis}

Cell apoptosis was analyzed by apoptosis detection kit (Solarbio, Beijing, China). In short, cells were collected and washed with pre-cooling PBS. Cells were suspended with binding buffer $(1 \mathrm{~mL})$. Cells were incubated with 
Annexin V-FITC and propidium iodide (PI), respectively. Cell apoptosis was analyzed using flow cytometry (BD Biosciences, San Diego, CA, USA).

\section{RNA Immunoprecipitation (RIP) Assay}

Magna RIP Kit (Millipore, Billerica, MA, USA) was employed to detect whether circASXL1 could form silenceinducing complexes with RNA. In short, cells were collected and lysed with RIP lysis buffer. Magnetic beads were incubated with anti-ago 2 or anti-IgG. Then, cell lysate was incubated with magnetic beads for $24 \mathrm{~h}$. Following magnetic beads were washed and mixed with protein K. Phenol, chloroform and isoamyl alcohol were used to purify RNA. Results were assessed by qRT-PCR.

\section{RNA Pull-Down Assay}

Biotinylated circASXL1 probe or Oligo probe obtained from GenePharma was transfected into cells, respectively, and cultured for $48 \mathrm{~h}$. Cells were lysed and lysate was incubated with streptavidin-coupled beads for $1 \mathrm{~h}$. Then, mixture was digested with protein K (Solarbio). Results were revealed by qRT-PCR.

\section{Dual-Luciferase Reporter Assay}

The binding sequence of miR-206 in circASXL1 was predicted by circBank online database. The wide-type (WT) circASXL1 contained the binding sequence of miR-206 and mutant (MUT) circASXL1 were sub-cloned into pmirGLO vector (Miaoling, Wuhan, China), and named as circASXL1-WT and circASXL1-MUT. Cell transfection was carried out using lipofectamine 2000 (Thermo Fisher). Luciferase activities were detected via Dual-Lucy Assay Kit (Solarbio). Ranilla Luciferase activity was employed as a reference of firefly luciferase activity.

\section{In vivo Tumor Formation Assay}

Charles River (Beijing, China) provided BALB/c nude mice (5-week older, $\mathrm{N}=18$ ). Mice were fed in pathogen-free condition. A549 cells $\left(5 \times 10^{6}\right)$ transfected with sh-circASXL1-1 or sh-NC were inoculated into the right side of the back of mice. And mice were treated with antagomiR-206 and antagomiR-NC by tail vein injection every 1 day for 3 days according to defined purpose. After 7 days, tumor volume was detected every 4 days. After 27 days, mice were euthanatized and tumor weight was determined. The effects between circASXL1 silencing and miR-206 downregulation on miR-206 expression were detected by qRT-PCR. The
Care and Use Committee of Jing Men NO.2 People's Hospital consented to this experiment. The National Institutes of Health guide for the care and use of Laboratory animals (NIH Publications No. 8023, revised 1978) was strictly followed in the present study.

\section{Statistical Analysis}

Figures in this study were made via GraphPad Prism 5.0 and image $\mathrm{J}$ software. Data was obtained based on 3 replicates. Data were presented as means \pm standard deviations (SD). Significant differences were assessed via twotailed Student's $t$-tests and one-way analysis of variance. $P<0.05$ was deemed statistically significant.

\section{Results}

\section{CircASXLI Expression Was Upregulated in NSCLC Tissues, Cells and Hypoxic NSCLC Cells}

In order to reveal the role of circASXL1 in NSCLC progression under hypoxia, the expression level of circASXL1 was determined in NSCLC after hypoxia treatment. QRT-PCR results firstly showed that circASXL1 expression was dramatically upregulated in NSCLC tissues and cells compared with control groups (Figure 1A and B). Then, results showed that circASXL1 expression was significantly increased in hypoxic A549 and H1299 cells when compared with normoxic cells (Figure 1C). Subsequently, we found that circASXL1 was 195 nucleotides in length and was generated by backsplicing between exons 2 and exons 3 of ASXL1 (Figure 1D). RNase $\mathrm{R}$ treatment assay showed that circASXL1 expression was not significantly changed after RNase R treatment, whereas GAPDH RNA (linear RNA) expression was dramatically downregulated in A549 and H1299 cells (Figure 1E), which suggested that circular RNA ASXL1 was more stable than linear RNA. In addition, cytoplasmic and nuclear RNA analysis of qRT-PCR indicated that circASXL1 was preferentially localized in the cytoplasm of A549 and H1299 cells (Figure 1F). These data showed that circASXL1 played a vital part in NSCLC progression.

\section{CircASXLI Knockdown Hindered Hypoxia-Induced Cell Proliferation, Migration and Invasion in A549 and HI299 Cells}

To explore the functional effects of circASXL1 knockdown on cell proliferation, migration and invasion under hypoxia, 
A

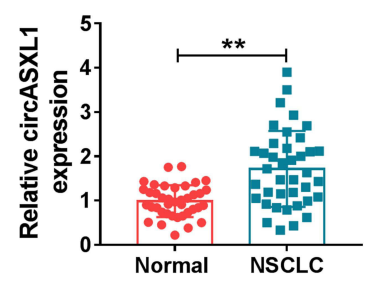

D

\section{ASXL1}

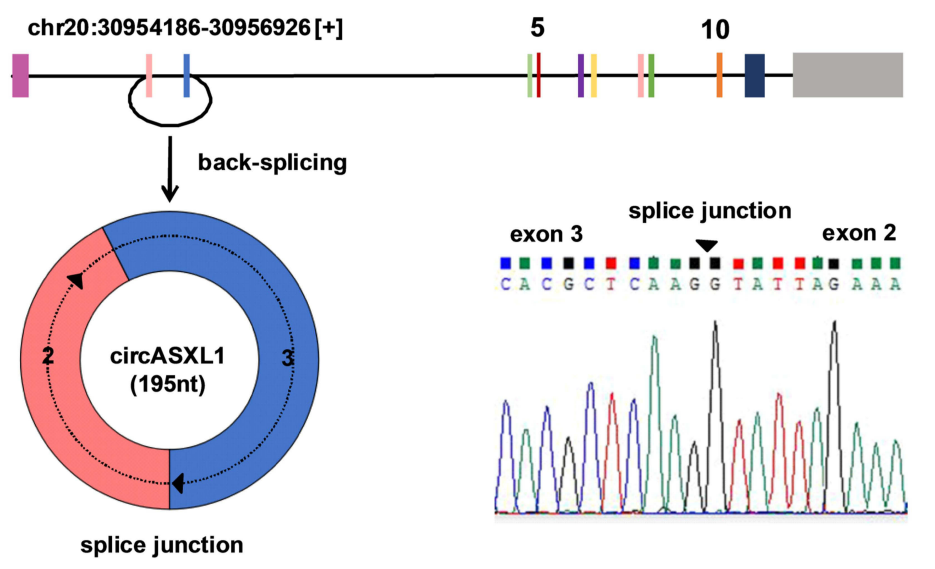

C

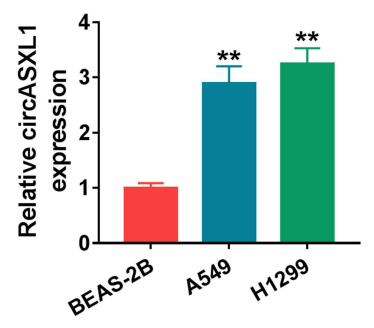

A549

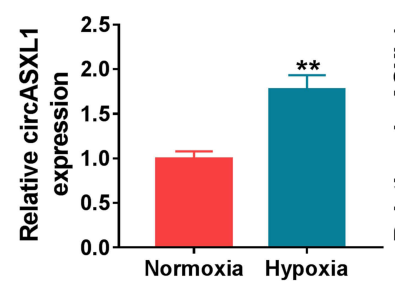

E
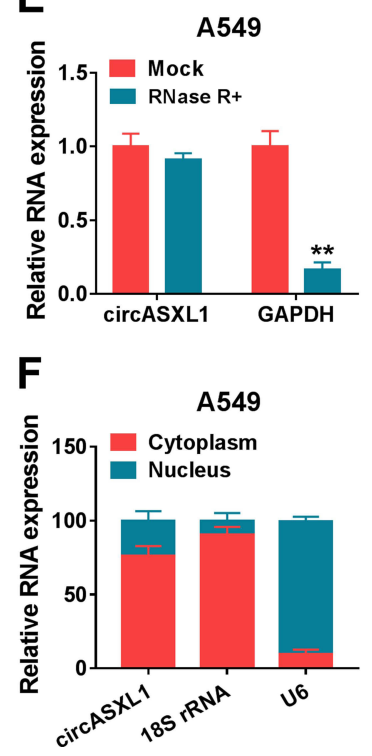

H1299
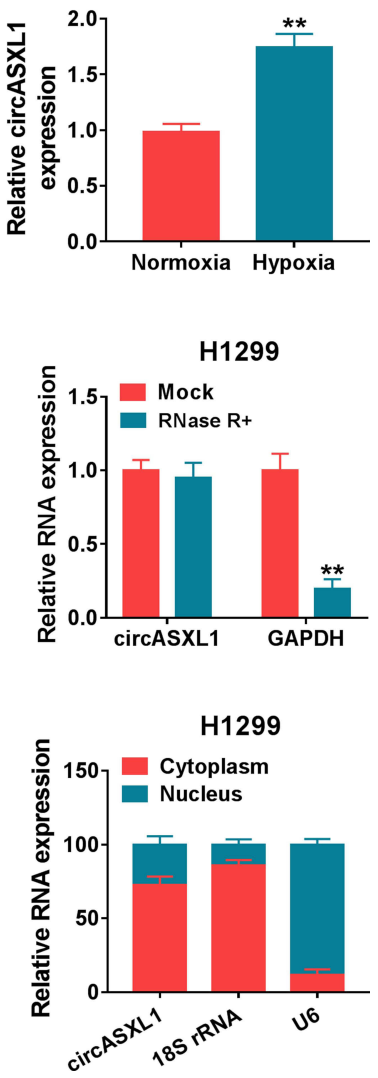

Figure I CircASXLI expression was increased in NSCLC tissues, cells and hypoxic cells. (A and B) CircASXLI expression was determined in NSCLC tissues, normal tissues, BEAS-2B, A549 and HI299 cells. (C) CircASXLI expression was detected in hypoxic and normoxic A549 and HI299 cells. (D) Schematic illustration showed that circASXLI was generated by back-splicing between exons 2 and exons 3 of ASXLI. (E) RNase R treatment assay revealed that circASXLI was more stable than linear RNA. (F) Cytoplasmic and nuclear RNA analysis explained that circASXLI mainly localized in the cytoplasm of A549 and HI299 cells. **P < $0.0 \mathrm{I}$.

the interfering plasmids of circASXL1 were built. QRT-PCR results demonstrated that circASXL1 expression was dramatically downregulated by sh-circASXL1-1 and shcircASXL1-2 (Figure 2A), and sh-circASXL1-1 was chosen for further study owing to its successful interfering efficiency. CCK-8 assay showed that the viability of A549 and H1299 cells was promoted under hypoxia conditions, whereas circASXL1 knockdown decreased this effect (Figure 2B). Cell colony formation assay unveiled that the colonyforming ability of A549 and H1299 cells was improved under hypoxia conditions, but circASXL1 deletion reversed this impact (Figure 2C). Transwell assay investigated that cell migratory and invasive abilities of A549 and H1299 cells were improved by hypoxia exposure, and circASXL1 silencing abolished these effects (Figure 2D and E). Furthermore, Western blot analysis revealed that the effects of circASXL1 knockdown on the expression of proliferation-related protein c-myc and metastasis-related proteins E-cadherin and Vimentin. Results showed that c-myc and Vimentin expression were increased, and E-cadherin expression was downregulated under hypoxia, whereas which were restored by circASXL1 absence (Figure 2F). All data showed that circASXL1 regulated hypoxia-mediated cell proliferation, migration and invasion in NSCLC.

\section{CircASXLI Knockdown Restrained Hypoxia-Mediated Inhibition Effects on DDP Sensitivity and Cell Apoptosis}

The effects between circASXL1 knockdown and hypoxia treatment on DDP resistance and cell apoptosis were continued to explore. CCK-8 assay demonstrated that IC50 value of DDP was upregulated under hypoxia conditions in A549 and H1299 cells, whereas circASXL1 knockdown hindered this effect (Figure 3A and B). Flow cytometry assay revealed that circASXL1 silencing restrained hypoxia-mediated inhibition effect on cell apoptosis after 12.5 $\mu \mathrm{M}$ DDP treatment in A549 and H1299 cells (Figure 3C). In addition, Western blot analysis also explained that circASXL1 knockdown restored hypoxia-mediated 


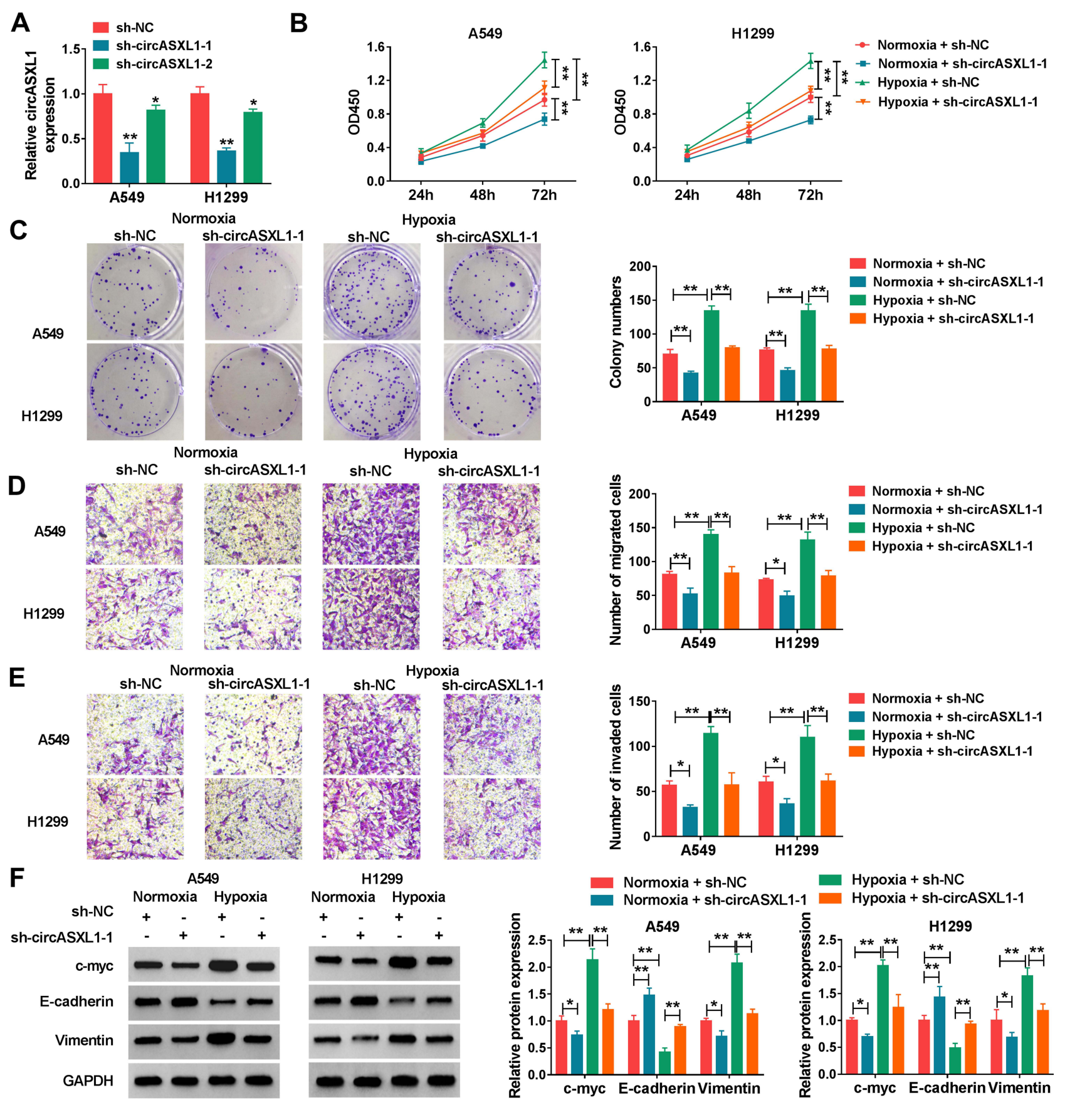

Figure 2 CircASXLI silencing abolished hypoxia-induced cell proliferation, migration and invasion in A549 and HI299 cells. (A) The interfering efficiency of sh-circASXLI-I and sh-circASXLI-2 was determined by qRT-PCR. (B) CCK-8 assay was employed to reveal the effect between circASXLI knockdown on cell viability after hypoxia treatment in A549 and HI299 cells. (C) Cell colony formation assay was carried out to display the impact between circASXLI silencing and hypoxia treatment on the colony-forming ability of A549 and HI299 cells. (D and E) The effects between circASXLI deletion and hypoxia treatment on cell migration and invasion in A549 and HI299 cells were determined by transwell assay. (F) Western blot was used to detect the effects between circASXLI knockdown and hypoxia exposure on the protein expression of c-myc, E-cadherin and Vimentin. $* P<0.05$ and $* * P<0.01$.

inhibition effect on apoptosis-related protein (Cleaved casp-3) and promotion effect on drug resistance-related protein (MDR1) after $12.5 \mu \mathrm{M}$ DDP treatment (Figure 3D). These results suggested that circASXL1 knockdown reversed hypoxia-mediated promotion effect on DDP resistance in NSCLC cells.

\section{CircASXLI Acted as a Sponge of miR-206} The regulatory mechanism of circASXL1 in NSCLC progression and DDP resistance after hypoxia treatment was revealed in this part. Whether circASXL1 had the ability to form silence-inducing complexes with miRNA was firstly demonstrated. RIP assay results showed that endogenous 

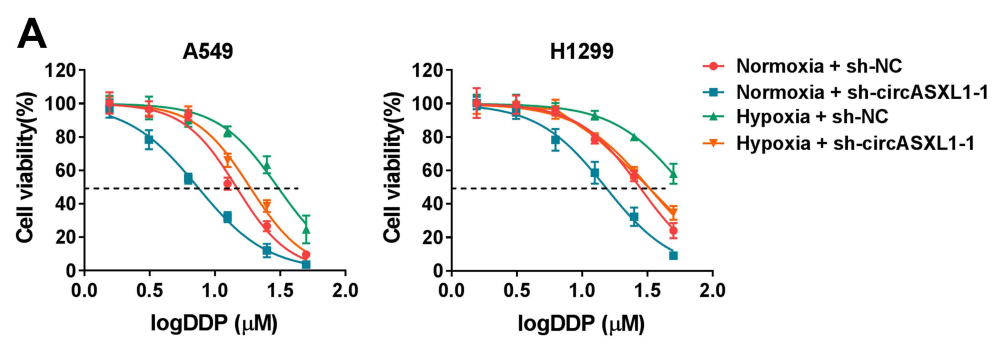

B

C

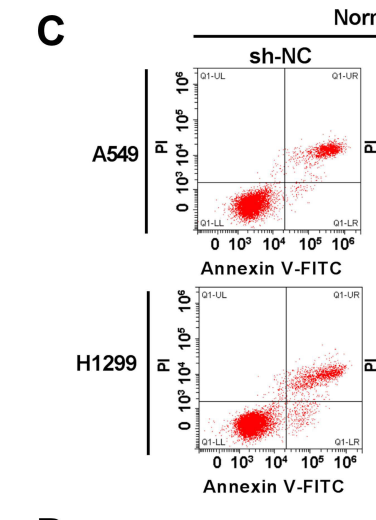

Normoxia

gDDP $(\mu \mathrm{M})$

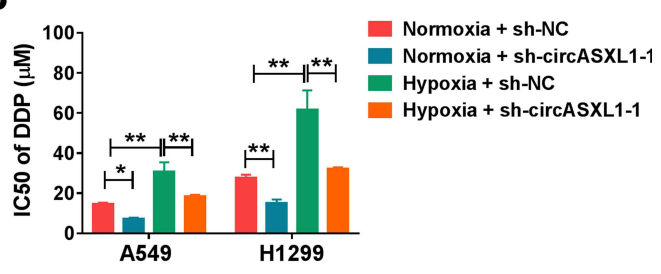

Hypoxia
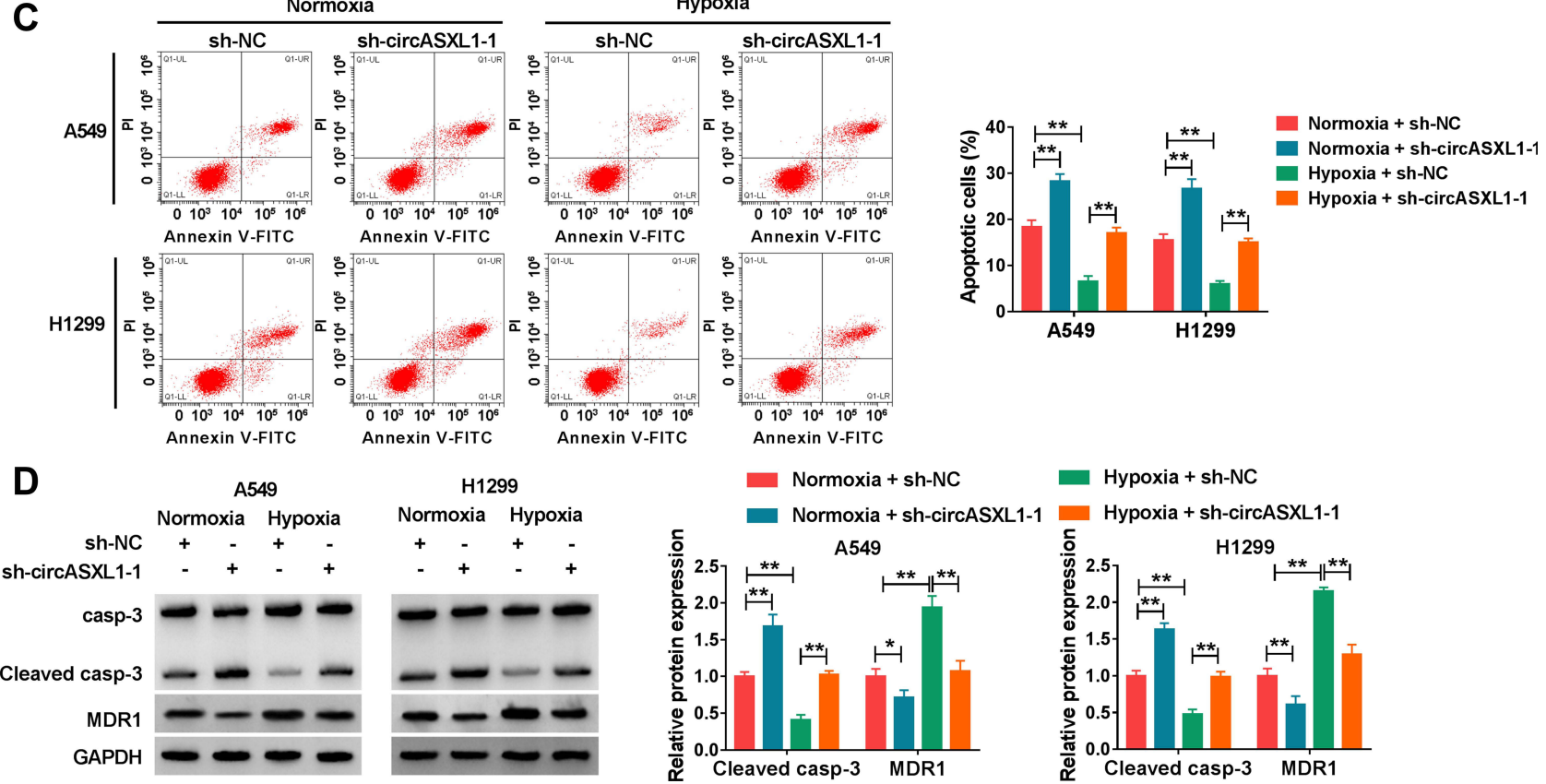

Figure 3 CircASXLI silencing restrained hypoxia-mediated inhibition effects on DDP sensitivity. (A and B) The effects between circASXLI deletion and hypoxia treatment on IC50 value and sensitivity of DDP in A549 and HI299 cells were determined by CCK-8 assay. (C) Flow cytometry analysis was employed to demonstrate the impacts between circASXLI absence and hypoxia exposure on cell apoptosis in A549 and HI299 cells. (D) The impacts between circASXLI silencing and hypoxia treatment on protein expression of Cleaved casp-3 and MDRI were revealed in A549 and HI299 cells by Western blot. $* P<0.05$ and $* * P<0.01$.

circASXL1 could bind to ago2 protein and was dramatically enriched in A549 and H1299 cells compared with cells transfected with sh-circASXL1-1 (Figure 4A), which suggested that circASXL1 could form silence-inducing complexes with small RNA. In order to determine the miRNAs associated with circASXL1 in A549 and H1299 cells, RNA pull-down assay was performed. Results showed that miR206 could be dramatically enriched by circASXL1 probe compared with that in Oligo probe group in A549 and H1299 cells (Figure 4B), and miR-206 was chosen as potential miRNA bound to circASXL1.

Then, circBank online database showed that circASXL1 contained the binding sites of miR-206 (Figure 4C). Dualluciferase reporter system was performed to identify the target relationship between miR-206 and circASXL1. QRTPCR results firstly verified that miR-206 expression was dramatically upregulated after miR-206 mimic transfection in A549 and H1299 cells (Figure 4D). And dual-luciferase reporter assay showed that the luciferase activity was significantly decreased after circASXL1-WT and miR-206 transfection in A549 and H1299 cells, whereas there was no obvious change in circASXL1-MUT+miR-206 group (Figure 4E). Subsequently, qRT-PCR results showed that miR-206 expression was dramatically upregulated by circASXL1 knockdown compared with control group (Figure 4F). QRT-PCR analysis also investigated that miR206 expression was markedly downregulated in NSCLC tissues and cells as compared as control groups (Figure 4G and $\mathrm{H}$ ). Meanwhile, miR-206 expression was downregulated after hypoxia treatment in A549 and H1299 cells (Figure 4I). All these data showed that circASXL1 was associated with miR-206 in A549 and H1299 cells. 

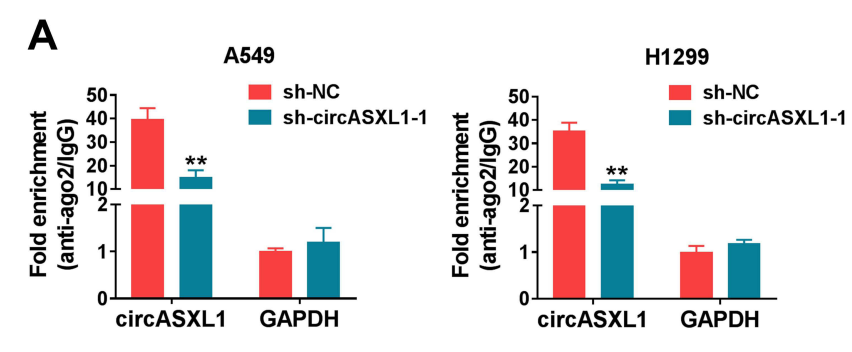

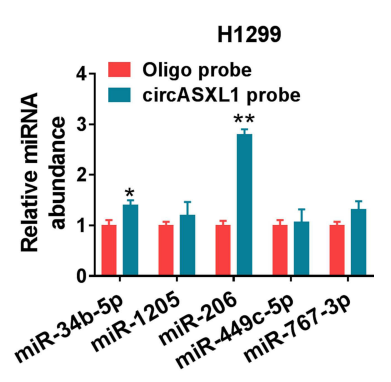

$E$

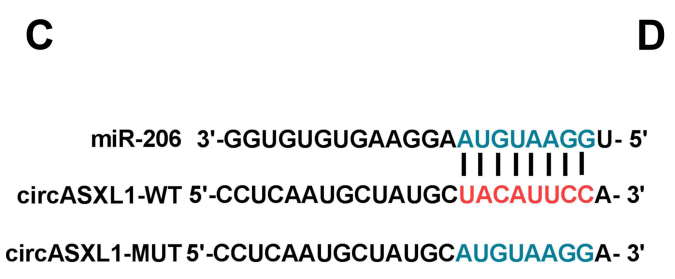

circASXL1-MUT 5'-CCUCAAUGCUAUGCAUGUAAGGA- 3'

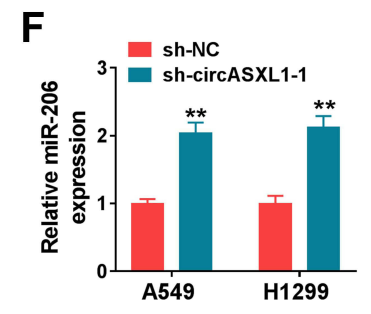

B
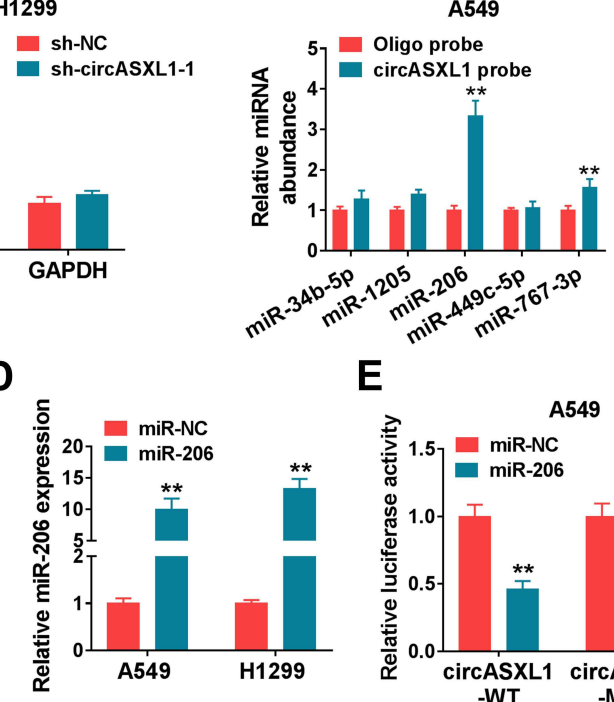
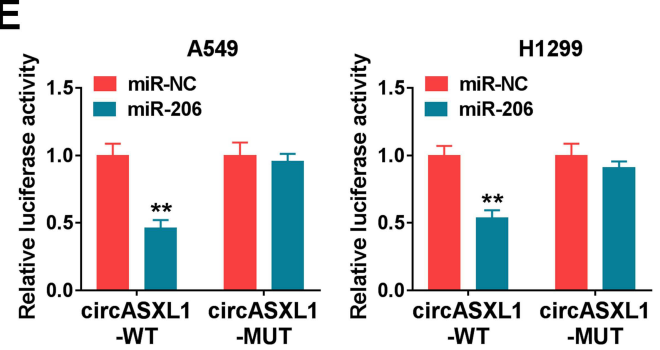
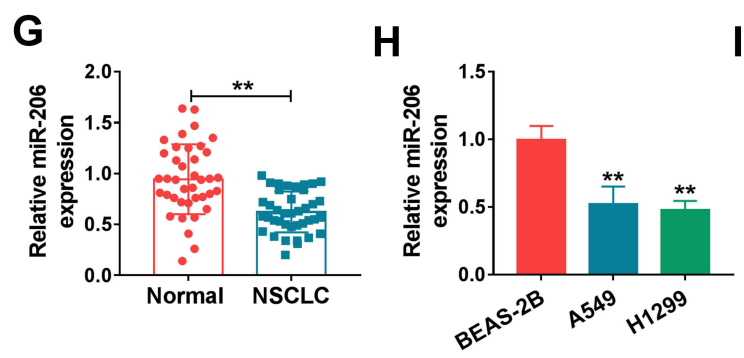

I

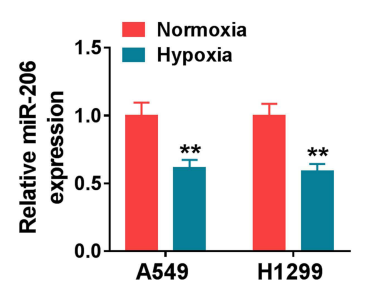

Figure 4 CircASXLI bound to miR-206 in A549 and HI299 cells. (A) RIP assay was performed to explain whether circASXLI could form silence-inducing complexes with miRNA in A549 and HI299 cells. (B) RNA pull-down assay was employed to determine the miRNAs associated with circASXLI. (C) CircBank online database was used to predict the binding sites between miR-206 and circASXLI. (D) MiR-206 expression was detected by qRT-PCR after miR-206 mimic transfection. (E) Dual-luciferase reporter assay was carried out to detect luciferase activities in A549 and HI299 cells. (F) The effect of circASXLI knockdown on miR-206 expression was revealed by qRT-PCR. (G and H) MiR-206 expression was determined by qRT-PCR in NSCLC tissues, NSCLC normal tissues, BEAS-2B cells, A549 cells and HI299 cells. (I) qRT-PCR was utilized to detect miR-206 expression in A549 and HI299 cells after normoxia or hypoxia treatment. $* P<0.05$ and $* * P<0.01$.

\section{CircASXLI Knockdown Restrained Hypoxia-Mediated Promotion Effects on Cell Proliferation, Migration and Invasion} by Sponging miR-206

The effects between circASXL1 and miR-206 on cell proliferation, migration and invasion under hypoxia treatment in NSCLC were continued to reveal. QRT-PCR was firstly employed to determine the interfering efficiency of miR-206 inhibitor, and results showed that miR-206 expression was dramatically downregulated after miR-206 inhibitor transfection (Figure 5A). Subsequently, CCK-8 assay showed that circASXL1 knockdown hindered cell viability and colony-forming ability under hypoxia in A549 and H1299 cells, whereas these effects were attenuated by miR-206 inhibitor (Figure 5B and C). Transwell assay demonstrated that circASXL1 silencing suppressed cell migratory and invasive abilities after hypoxia treatment, which were decreased after miR-206 inhibitor transfection (Figure 5D and E). In addition, Western blot investigated that circASXL1 knockdown downregulated the protein expression of c-myc and Vimentin and upregulated E-cadherin protein expression after hypoxia exposure; whereas these effects were abolished by miR-206 inhibitor (Figure 5F). All the above data explained that circASXL1 regulated hypoxia-induced cell proliferation, migration and invasion by associating with miR-206.

\section{CircASXLI Knockdown Reversed Hypoxia-Mediated Inhibition Effects on DDP Sensitivity and Cell Apoptosis by} Binding to miR-206

The effects between circASXL1 silencing and miR-206 inhibitor on DDP resistance and cell apoptosis after hypoxia exposure were continued to explore. CCK-8 assay demonstrated that circASXL1 deletion repressed DDP resistance under hypoxia in A549 and H1299 cells, whereas miR-206 


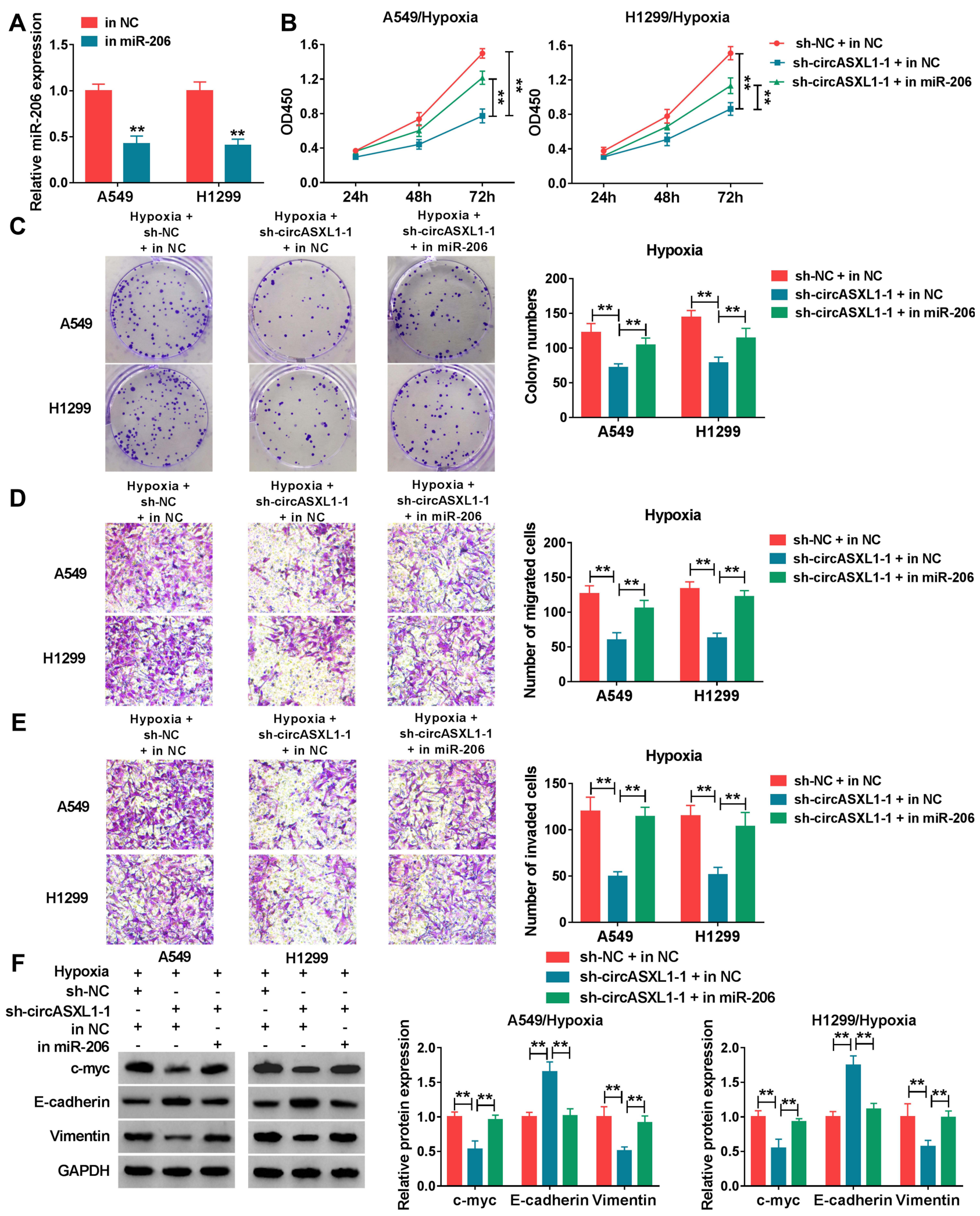

Figure 5 CircASXLI deletion decreased hypoxia-induced cell proliferation, migration and invasion by binding to miR-206. (A) The inferring efficiency of miR-206 inhibitor was determined by qRT-PCR. (B and C) The effects between circASXLI knockdown and miR-206 inhibitor on hypoxia-induced cell proliferation were illustrated by CCK-8 assay. (D and E) Transwell assay was performed to disclose the impacts between miR-206 inhibitor and circASXLI silencing on hypoxia-mediated cell migration and invasion.

(F) The effects between circASXLI deletion and miR-206 inhibitor on the protein expression of c-myc, E-cadherin and Vimentin after hypoxia treatment were determined by Western blot. $* * P<0.01$. 
inhibitor restored this impact (Figure 6A and B). Flow cytometry analysis showed that circASXL1 knockdown induced cell apoptosis after hypoxia exposure; however, which was restrained by miR-206 inhibitor (Figure 6C). Meanwhile, Western blot analysis illustrated that sh-circASXL1-1 upregulated Cleaved casp-3 expression and downregulated MDR1 protein expression under hypoxia treatment, and these effects were abolished by miR-206 inhibitor (Figure 6D). These results suggested that circASXL1 deletion hindered hypoxiamediated effects on DDP resistance and cell apoptosis by associating with miR-206 in A549 and H1299 cells.

\section{CircASXLI Silencing Downregulated HIF- I $\alpha$ Expression by Regulating miR-206 Under Hypoxia}

Based on HIF-1 $\alpha$ acting as an important regulator of adaptive response to hypoxia, whether circASXL1 affected HIF$1 \alpha$ expression by binding to miR-206 was further explored. Results firstly illustrated that HIF-1 $\alpha$ protein expression was dramatically upregulated after hypoxia treatment, whereas circASXL1 silencing attenuated this effect (Supplementary Figure S1A). Subsequently, data showed that miR-206
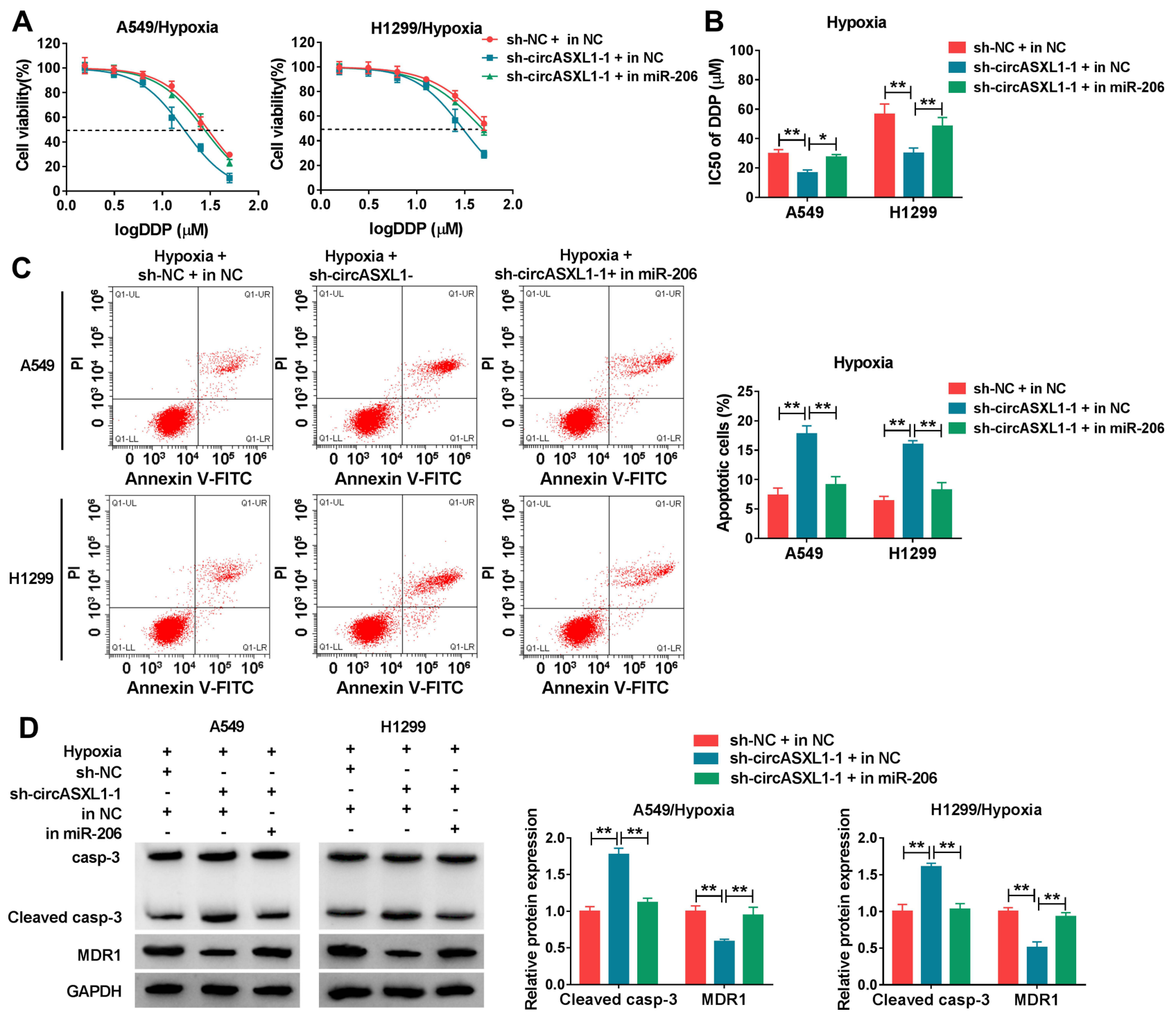

Figure 6 CircASXLI silencing promoted DDP sensitivity and cell apoptosis after hypoxia treatment by sponging miR-206. (A and B) The effects between circASXLI knockdown and miR-206 inhibitor on DDP resistance after hypoxia treatment were revealed by CCK-8 assay. (C) Flow cytometry analysis was performed to demonstrate the impacts between circASXLI silencing and miR-206 deletion on hypoxia-mediated cell apoptosis. (D) The effects between circASXLI knockdown and miR-206 inhibitor on hypoxia-mediated expression of Cleaved casp-3 and MDRI were revealed by Western blot. $* P<0.05$ and $* * P<0.01$. 
inhibitor restrained the inhibition impact of circASXL1 absence on the protein expression of HIF-1 $\alpha$ under hypoxia (Supplementary Figure S1B). The above data demonstrated that circASXL1 could modulate HIF-1 $\alpha$ expression through absorbing miR-206 under hypoxia in NSCLC.

\section{CircASXLI Knockdown Repressed Tumor Growth in vivo by Associating with miR-206}

Whether circASXL1 regulated tumor formation in vivo by binding to miR-206 was continued to illustrate. Results showed that circASXL1 silencing reduced tumor volume and weight, whereas miR-206 absence impaired these impacts (Figure 7A-C). Furthermore, the effects between circASXL1 knockdown and miR-206 downregulation on miR-206 expression in vivo were demonstrated. QRT-PCR results presented that circASXL1 absence upregulated miR-206 expression; however, miR-206 inhibitor attenuated this influence (Figure 7D). All results showed that
circASXL1 knockdown suppressed tumor growth by absorbing miR-206 in vivo.

\section{Discussion}

NSCLC is a primary cause of carcinoma-linked mortality and DDP is a main drug in NSCLC therapy. ${ }^{22}$ But the chemoresistance of DDP has limited its positive effect to NSCLC. CircRNA has been revealed to be closely linked to cancer progression and DDP resistance. ${ }^{23,24}$ Herein, the effects and mechanism of hypoxia treatment regulated by circASXL1 on DDP resistance were illustrated.

Previous study illustrated that circASXL1 expression was upregulated in bladder cancer cells and was negatively correlated with survival rate. $^{25}$ From our findings, circASXL1 was revealed for the first time to be upregulated in NSCLC tissues, cells and hypoxia-mediated NSCLC cells. In addition, circASXL1 knockdown was revealed to inhibit DDP resistance, cell proliferation, migration and invasion, whereas induced cell apoptosis
A

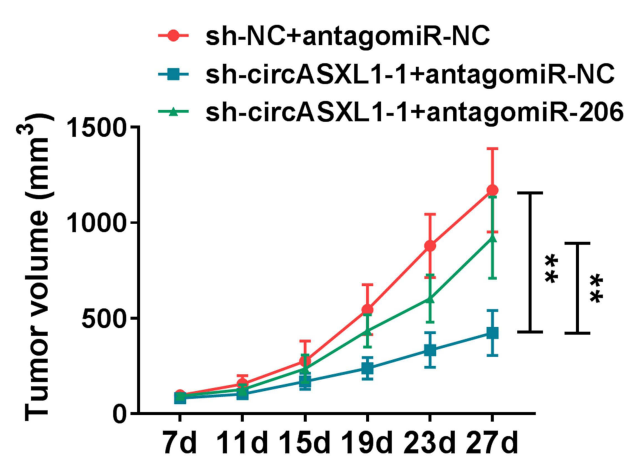

B

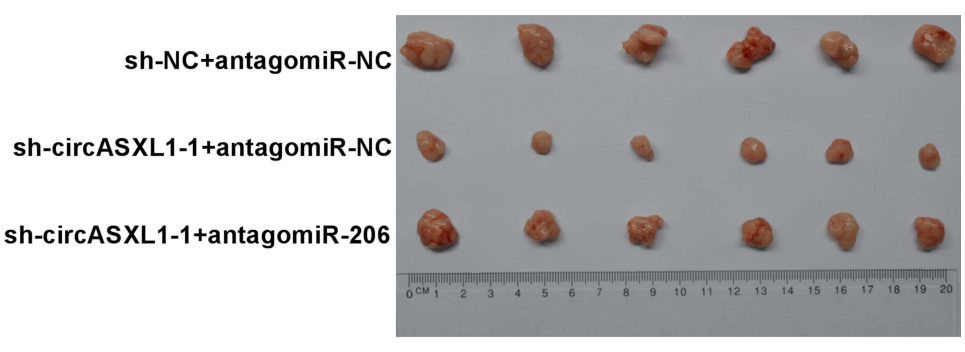

C
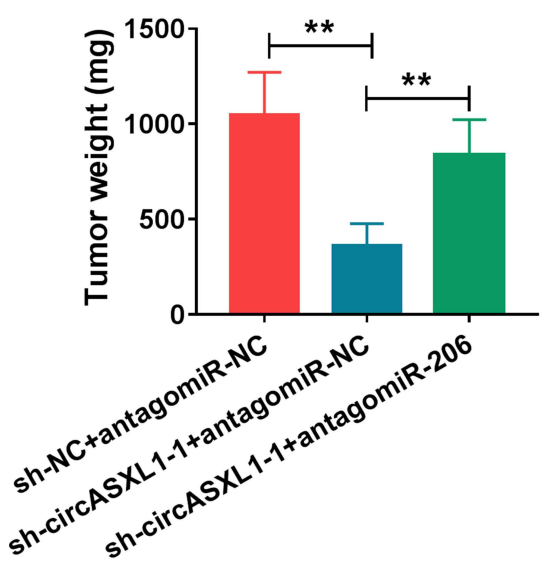

D

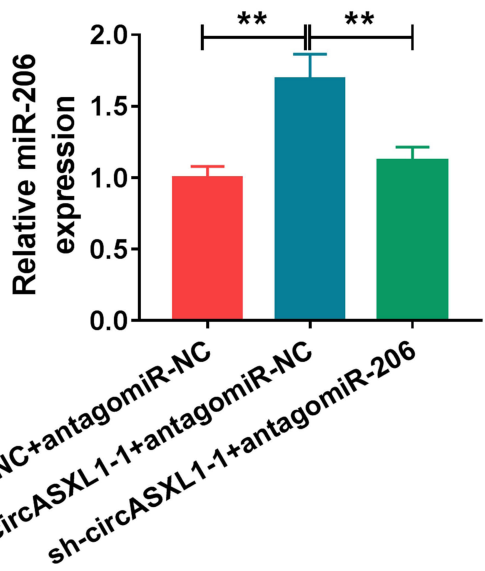

Figure 7 CircASXLI silencing inhibited tumor growth in vivo by upregulating miR-206 expression. (A-C) The effects between circASXLI knockdown and miR-206 inhibitor on tumor volume and weight were determined in vivo. (D) qRT-PCR was performed to detect the effects between circASXLI knockdown and miR-206 downregulation on the expression of circASXLI and miR-206 in vivo. ${ }^{* * P}<0.01$. 
after hypoxia treatment in NSCLC. These results suggested that circASXL1 knockdown attenuated the effects of hypoxia treatment on NSCLC progression. Furthermore, circASXL1 was investigated to act as a sponge of miR-206.

Hypoxia treatment was revealed to be linked to cancer progression. Ren et al demonstrated that cell migratory and invasive abilities were improved under hypoxia in breast cancer. ${ }^{26}$ Yin et al indicated that hypoxia treatment promoted DDP resistance and cell proliferation, and repressed cell apoptosis. ${ }^{27} \mathrm{Su}$ et al explained that hypoxia treatment accelerated cell migratory and invasive abilities in glioma. ${ }^{28}$ These research indicated that hypoxia treatment accelerated cancer progression. Similarly, in our study, hypoxia promoted cell migratory and invasive abilities in NSCLC. We also found hypoxia exposure promoted cell proliferation and DDP resistance, and repressed cell apoptosis in NSCLC.

MiR-206 was reported to inhibit NSCLC progression. Zhao et al indicated miR-206 inhibited cell viability, colonyforming ability and metastasis in NSCLC. ${ }^{29}$ MiR-206 also participated in regulation in cancer progression under hypoxia. Tan et al unveiled miR-206 expression was downregulated in thyroid carcinoma tissues, cells and hypoxia-mediated thyroid carcinoma, and its inhibitor restrained the inhibition effects of nuclear paraspeckle assembly transcript 1 on hypoxiamediated cell migration and invasion anaplastic thyroid carcinoma cells, ${ }^{30}$ which meant that miR-206 suppressed cell migration and invasion under hypoxia. We also disclosed that miR-206 expression was decreased in NSCLC tissues, cells and hypoxia-mediated NSCLC cells, and inhibited cell migratory and invasive abilities after hypoxia exposure in NSCLC. In addition, miR-206 was shown to inhibit cell proliferation and DDP resistance, whereas induced cell apoptosis under hypoxia. In vivo tumor formation assay presented that circASXL1 modulated tumor growth via absorbing miR-206 in vivo. The above data investigated that circASXL1 silencing repressed hypoxia-induced DDP resistance and NSCLC development by sponging miR-206.

HIFs are vital regulators of adaptive response to hypoxia, ${ }^{31}$ and HIF-1 $\alpha$, one of them, is reported to be highly expressed in various cancers. ${ }^{32,33}$ Thus, whether circASXL1 could regulate HIF-1 $\alpha$ expression under hypoxia was further explored. Results showed that circASXL1 silencing could attenuate the promotion effect of hypoxia exposure on HIF$1 \alpha$ protein expression. Of note, miR-206 downregulation restored the inhibition effect of circASXL1 on HIF-1 $\alpha$ expression. These findings illustrated circASXL1 could modulate HIF-1 $\alpha$ expression by absorbing miR-206 under hypoxia in NSCLC, implying circASXL1 might modulate hypoxia-induced DDP resistance and NSCLC process by controlling HIF-1 $\alpha$ expression.

Collectively, circASXL1 expression was dramatically upregulated, and miR-206 was significantly downregulated in NSCLC tissues, cells and hypoxia-mediated NSCLC cells. CircASXL1 knockdown restrained hypoxia-induced DDP resistance and NSCLC progression by sponging miR-206 in NSCLC cells. In addition, circASXL1 silencing downregulated HIF-1 $\alpha$ expression by regulating miR-206, implying circASXL1 might modulate hypoxia-induced DDP resistance and NSCLC process by controlling HIF-1 $\alpha$ expression, which laid a foundation for further revealing the mechanism of circASXL2 in regulating hypoxia-induced DDP resistance and NSCLC progression. These findings also provide a new insight for the study of DDP in treating NSCLC.

\section{Funding}

This work was approved by the Outstanding Young and Middle-aged Scientific and Technological Innovation Team Program of Colleges and Universities in Hubei Province (No.T201819).

\section{Disclosure}

The authors declare that they have no financial and/or other conflicts of interest in this work.

\section{References}

1. Siegel RL, Miller KD, Jemal A. Cancer statistics, 2019. CA Cancer J Clin. 2019;69(1):7-34. doi:10.3322/caac.21551

2. Fischer C, Leithner K, Wohlkoenig C, et al. Panobinostat reduces hypoxia-induced cisplatin resistance of non-small cell lung carcinoma cells via HIF-1 $\alpha$ destabilization. Mol Cancer. 2015;14(1):4. doi:10.1186/1476-4598-14-4

3. Guo J, Jin D, Wu Y, et al. The miR 495-UBE2C-ABCG2/ERCC1 axis reverses cisplatin resistance by downregulating drug resistance genes in cisplatin-resistant non-small cell lung cancer cells. EBioMedicine. 2018;35:204-221. doi:10.1016/j.ebiom.2018.08.001

4. Torre LA, Bray F, Siegel RL, et al. Global cancer statistics, 2012. CA Cancer J Clin. 2015;65(2):87-108. doi:10.3322/caac.21262

5. Pirker R. Adjuvant chemotherapy in patients with completely resected non-small cell lung cancer. Transl Lung Cancer Res. 2014;3 (5):305-310. doi:10.3978/j.issn.2218-6751.2014.09.13

6. Li K, Guo J, Wu Y, et al. Suppression of YAP by DDP disrupts colon tumor progression. Oncol Rep. 2018;39(5):2114-2126. doi:10.3892/ or.2018.6297

7. Muz B, De La Puente P, Azab F, et al. The role of hypoxia in cancer progression, angiogenesis, metastasis, and resistance to therapy. Hypoxia. 2015;3:83-92. doi:10.2147/HP.S93413

8. Chou $\mathrm{CW}$, Wang $\mathrm{CC}, \mathrm{Wu} \mathrm{CP}$, et al. Tumor cycling hypoxia induces chemoresistance in glioblastoma multiforme by upregulating the expression and function of ABCB1. Neuro Oncol. 2012;14 (10):1227-1238. doi:10.1093/neuonc/nos195 
9. Flamant L, Roegiers E, Pierre M, et al. TMEM45A is essential for hypoxia-induced chemoresistance in breast and liver cancer cells. BMC Cancer. 2012;12(1):391. doi:10.1186/1471-2407-12-391

10. Ebbesen KK, Kjems J, Hansen TB. Circular RNAs: identification, biogenesis and function. Biochim Biophys Acta. 2016;1859 (1):163-168. doi:10.1016/j.bbagrm.2015.07.007

11. Zhu LP, He YJ, Hou JC, et al. The role of circRNAs in cancers. Biosci Rep. 2017;37(5). doi:10.1042/BSR20170750

12. Kristensen LS, Hansen TB, Venø MT, et al. Circular RNAs in cancer: opportunities and challenges in the field. Oncogene. 2018;37 (5):555-565. doi:10.1038/onc.2017.361

13. Li M, Liu Y, Liu J, et al. Circ_0006332 promotes growth and progression of bladder cancer by modulating MYBL2 expression via miR-143. Aging. 2019;11(22):10626-10643. doi:10.18632/ aging.102481

14. Zhang C, Li Y, Zhao W, et al. Circ-PGAM1 promotes malignant progression of epithelial ovarian cancer through regulation of the miR-542-3p/CDC5L/PEAK1 pathway. Cancer Med. 2020;9 (10):3500-3521. doi:10.1002/cam4.2929

15. $\mathrm{Xu} \mathrm{G}, \mathrm{Li} \mathrm{M}, \mathrm{Wu} \mathrm{J}$, et al. Circular RNA circNRIP1 sponges microRNA-138-5p to maintain hypoxia-induced resistance to 5-Fluorouracil Through HIF-1 $\alpha$-dependent glucose metabolism in gastric carcinoma. Cancer Manag Res. 2020;12:2789-2802. doi:10.2147/CMAR.S246272

16. Huang M-S, Liu J-Y, Xia X-B, et al. Hsa_circ_0001946 inhibits lung cancer progression and mediates cisplatin sensitivity in non-small cell lung cancer via the nucleotide excision repair signaling pathway. Front Oncol. 2019;9:508. doi:10.3389/fonc.2019.00508

17. Gebert LFR, Macrae IJ. Regulation of microRNA function in animals. Nat Rev Mol Cell Biol. 2019;20(1):21-37. doi:10.1038/ s41580-018-0045-7

18. $\mathrm{Wu} \mathrm{W,} \mathrm{Xi} \mathrm{W,} \mathrm{Li} \mathrm{H}$, et al. Circular RNA circ-ACACA regulates proliferation, migration and glycolysis in non-small-cell lung carcinoma via miR-1183 and PI3K/PKB pathway. Int J Mol Med. 2020;45 (6):1814-1824. doi:10.3892/ijmm.2020.4549

19. Gao P, Wang H, Yu J, et al. miR-3607-3p suppresses non-small cell lung cancer (NSCLC) by targeting TGFBR1 and CCNE2. PLoS Genet. 2018;14(12):e1007790. doi:10.1371/journal.pgen.1007790

20. Zhang Y-J, Xu F, Zhang Y-J, et al. miR-206 inhibits non small cell lung cancer cell proliferation and invasion by targeting SOX9. Int J Clin Exp Med. 2015;8(6):9107-9113.

21. Chen Q-Y, Jiao D-M, Wang J, et al. miR-206 regulates cisplatin resistance and EMT in human lung adenocarcinoma cells partly by targeting MET. Oncotarget. 2016;7(17):24510-24526. doi:10.18632/ oncotarget.8229
22. Oliver TG, Mercer KL, Sayles LC, et al. Chronic cisplatin treatment promotes enhanced damage repair and tumor progression in a mouse model of lung cancer. Genes Dev. 2010;24(8):837-852. doi:10.1101/ gad. 1897010

23. Yang F, Hu A, Li D, et al. Circ-HuR suppresses HuR expression and gastric cancer progression by inhibiting CNBP transactivation. Mol Cancer. 2019;18(1):158. doi:10.1186/s12943-019-1094-Z

24. Xue M, Li G, Fang X, et al. hsa_circ_0081143 promotes cisplatin resistance in gastric cancer by targeting miR-646/CDK6 pathway. Cancer Cell Int. 2019;19(1):25. doi:10.1186/s12935-019-0737-x

25. Tang G, Xie W, Qin C, et al. Expression of circular RNA circASXL1 correlates with TNM classification and predicts overall survival in bladder cancer. Int J Clin Exp Pathol. 2017;10(8):8495-8502.

26. Ren S, Liu J, Feng Y, et al. Knockdown of circDENND4C inhibits glycolysis, migration and invasion by up-regulating $\mathrm{miR}-200 \mathrm{~b} / \mathrm{c}$ in breast cancer under hypoxia. J Exp Clin Cancer Res. 2019;38(1):388. doi:10.1186/s13046-019-1398-2

27. Yin X, Liao Y, Xiong W, et al. Hypoxia-induced lncRNA ANRIL promotes cisplatin resistance in retinoblastoma cells through regulating ABCG2 expression. Clin Exp Pharmacol Physiol. 2020;47 (6):1049-1057. doi:10.1111/1440-1681.13279

28. Su H, Zou D, Sun Y, et al. Hypoxia-associated circDENND2A promotes glioma aggressiveness by sponging miR-625-5p. Cell Mol Biol Lett. 2019;24(1):24. doi:10.1186/s11658-019-0149-x

29. Zhao L, Zhang X, Shi Y, et al. LncRNA SNHG14 contributes to the progression of NSCLC through miR-206/G6PD pathway. Thoracic Cancer. 2020;11(5):1202-1210. doi:10.1111/1759-7714.13374

30. Tan X, Wang P, Lou J, et al. Knockdown of lncRNA NEAT1 suppresses hypoxia-induced migration, invasion and glycolysis in anaplastic thyroid carcinoma cells through regulation of miR-206 and miR-599. Cancer Cell Int. 2020;20(1):132. doi:10.1186/s12935-02001222-X

31. Hu X, Lin J, Jiang M, et al. HIF-1 $\alpha$ promotes the metastasis of esophageal squamous cell carcinoma by targeting SP1. J Cancer. 2020;11(1):229-240. doi:10.7150/jca.35537

32. Peerlings J, Van De Voorde L, Mitea C, et al. Hypoxia and hypoxia response-associated molecular markers in esophageal cancer: a systematic review. Methods. 2017;130:51-62. doi:10.1016/j. ymeth.2017.07.002

33. Berezowska S, Galván JA, Langer R, et al. Glycine decarboxylase and HIF-1 $\alpha$ expression are negative prognostic factors in primary resected early-stage non-small cell lung cancer. Virchows Arch. 2017;470(3):323-330. doi:10.1007/s00428-016-2057-z

\section{Publish your work in this journal}

Cancer Management and Research is an international, peer-reviewed open access journal focusing on cancer research and the optimal use of preventative and integrated treatment interventions to achieve improved outcomes, enhanced survival and quality of life for the cancer patient.
The manuscript management system is completely online and includes a very quick and fair peer-review system, which is all easy to use. Visit http://www.dovepress.com/testimonials.php to read real quotes from published authors. 